\title{
Nonlinear Simulation of Mixers for Assessing System-Level Performance
}

\author{
Nuno B. Carvalho, ${ }^{1}$ José C. Pedro, ${ }^{1}$ Wonhoon Jang, ${ }^{2}$ Michael B. Steer ${ }^{2}$ \\ ${ }^{1}$ Instituto de Telecomunicacoes-Universidade de Aveiro, Aveiro, Portugal \\ ${ }^{2}$ North Carolina State University, Raleigh, North Carolina
}

Received 4 October 2004; accepted 23 December 2004

\begin{abstract}
This article deals with nonlinear simulation methods intended to evaluate the impact of mixer nonidealities on the performance of a wireless system. Behavioral models capable of accurately describing the mixer's nonlinear dynamic features at the system level are currently unavailable. The possibility of using alternative circuit analysis techniques to reach this goal is discussed. After a brief review of existing mixer analysis methods, the focus is directed to the techniques amenable to efficiently handling periodic carriers modulated by complex stochastic signals. In particular, it is shown how multi-envelope transient methods coupled with a three-dimensional harmonic-balance engine can model a nonlinear dynamic mixer excited by a modulated RF signal accompanied by a strong adjacent channel interferer and with a local oscillator corrupted by phase noise. (c) 2005 Wiley Periodicals, Inc. Int J RF and Microwave CAE 15: 350-361, 2005.
\end{abstract}

Keywords: mixers; nonlinear systems; harmonic-balance; simulation techniques

\section{INTRODUCTION}

Mixers constitute key elements in superheterodyne transceivers for wireless systems, but are the primary sources of nonlinear distortion. In contrast to amplifiers, for example, in which nonlinearity is an undesirable side effect, a nonlinear process is central to mixer operation. Indeed, eliminating mixer nonlinearity as a means to reduce signal distortion would have the catastrophic effect of increasing conversion loss without limit. This weird situation can be related to the paradoxical fact that, although an ideal mixer is one device that generates an output (the intermediate frequency, IF) that is an exact (linear) frequencyshifted replica of the input (the radio frequency, RF),

\footnotetext{
Correspondence to: Nuno B. de Carvalho; email: nborges@ieee.org.

DOI 10.1002/mmce.20091

Published online 9 May 2005 in Wiley InterScience (www. interscience.wiley.com)
}

frequency translation is in essence a nonlinear process.

Mixers are characterized for nonlinear distortion by specifications including second- or third-order intercept points [1]. Those characteristics associated with the gain conversion are then used to extract a cubic polynomial model, i.e., a mildly nonlinear and memoryless behavioral representation that is still the one most widely employed for system analysis and design. Unfortunately, this oversimplified black-box model does not account for other extremely important mixer nonidealities such as saturation, linear or nonlinear dynamics, additive noise, local oscillator (LO) phase noise, and desensitization and cross-modulation induced by a strong adjacent channel (AC) interferer. Indeed, despite initial steps having been taken to develop accurate nonlinear and dynamic black-box models, behavioral modeling of mixers is not yet at a stage capable of responding to the needs of system designers. Thus, when more accurate analysis data is sought, engineers are forced to increase the descrip- 
tion detail and simulate the mixer at the equivalent circuit level. Even that is generally difficult, since most nonlinear circuit analysis methods are inadequate to treat a nonlinear dynamic regime with several carriers modulated by complex stochastic envelopes.

In this article we begin by reviewing existing circuit simulation methods, comparing their ability to efficiently handle complex multicarrier excitations. For example, we explain how the harmonic-balance (HB) technique [1-6], initially conceived for simple sinusoidal excitations, can now be used for more complex stimuli. It may be readily applied to two- or three-tone excitations when based on the Multidimensional Discrete Fourier Transform (MDFT) [5], or to more general multitones if coupled with an artificial frequency mapping (AFM) scheme [4, 6]. Nevertheless $\mathrm{HB}$, being a frequency-domain method, requires periodicity, or at least quasi-periodicity, from the applied stimulus. Time-varying Volterra series [2] could, in principle, be used to circumvent the difficulties imposed by aperiodic envelopes, but would then restrict the mixer operation to mildly nonlinear regimes.

Finally, the method that seems most promising is the envelope transient harmonic-balance technique [7-12]. The method assumes that all carriers and their complex modulation envelopes evolve in independent time scales. In particular, advantage is taken of the periodic nature of the carriers by treating them with $\mathrm{HB}$ in frequency domain, but still respects the aperiodic nature of the envelopes, evaluating them in a time-step by time-step process [13].

Considering a particular GaAs MESFET-based active single-ended mixer circuit, several mixer simulation examples are then shown in the final part. Starting with the simplest linear regime of a modulated RF input signal, and a pure LO sinusoid, the examples grow in complexity up to the above-referred case in that a modulated RF accompanied by a strong AC interferer are converted with an LO that is modulated by phase-noise.

\section{MIXER SIMULATION AT THE ENVELOPE/CIRCUIT LEVEL}

In order to present the available mixer simulation techniques with a practical perspective, let us consider the simple circuit of Figure 1, to which the different methods will be applied.

Despite its simplicity, this circuit includes memoryless nonlinearity, embodied in the nonlinear current source, $i_{N L}(t)$, and also nonlinear dynamic behavior, represented by the nonlinear charge $q_{N L}(t)$.

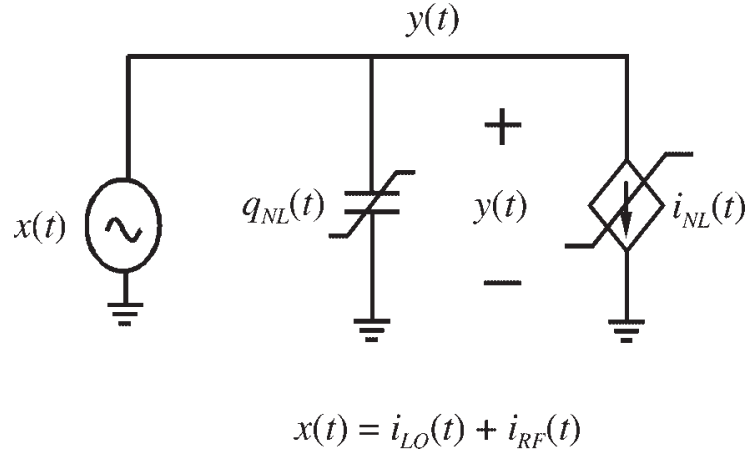

Figure 1. Nonlinear dynamic circuit example for the illustration of mixer simulation techniques.

Using nodal analysis, the mathematical model of this circuit becomes the following ordinary differential equation (ODE):

$$
\frac{d q_{N L}[\mathbf{y}(t)]}{d t}+i_{N L}[\mathbf{y}(t)]=\mathbf{x}(t)
$$

Here, $\mathbf{x}(t)$ is the input excitation vector (in a mixer, the $\mathrm{RF}-x_{R F}(t)$, and $\mathrm{LO}-x_{L O}(t)$, signals $), \mathbf{y}(t)$ is the state or output response vector (RF, LO, IF, and all other spurious mixing products), $q_{N L}[$.] is an algebraic nonlinear charge or flux function of the state vector, and finally, $i_{N L}[$.$] is an algebraic current or voltage of the$ same vector.

In typical telecommunication applications, the LO is a periodic signal (generally sinusoidal) which can be expanded in the following Fourier series:

$$
x_{L O}(t)=\sum_{k=-K}^{K} X_{l o}(k) e^{j k \omega_{0} t}
$$

On the contrary, the RF signal is a high-frequency carrier of frequency $\omega_{R F}$ modulated in amplitude - $r(t)$ - and phase - $\phi(t)$ :

$$
x_{R F}(t)=r(t) \cos \left[\omega_{R F} t+\phi(t)\right] \text {. }
$$

This is modulated by a typically aperiodic complex envelope, $x_{m}(t)$ defined as:

$$
x_{m}(t)=r(t) e^{j \phi(t)} .
$$

Considering that the transmitted RF signal, the generated LO, and the complex modulation envelope are all uncorrelated, we can imagine that the mixer operates as if it were driven in three artificial and independent time scales, $\tau_{1}, \tau_{2}$, and $\tau_{3}$. Thus, both the 
excitation and the state vectors become dependent on these new artificial time scales:

$$
\mathbf{x}(t) \rightarrow \mathbf{x}\left(\tau_{1}, \tau_{2}, \tau_{3}\right) ; \quad \mathbf{y}(t) \rightarrow \mathbf{y}\left(\tau_{1}, \tau_{2}, \tau_{3}\right)
$$

Accordingly, the original circuit ODE becomes a multi-rate partial differential equation (MPDE) [11] in these three variables:

$$
\begin{aligned}
& \frac{\partial q_{N L}\left[\mathbf{y}\left(\tau_{1}, \tau_{2}, \tau_{3}\right)\right]}{\partial \tau_{1}}+\frac{\partial q_{N L}\left[\mathbf{y}\left(\tau_{1}, \tau_{2}, \tau_{3}\right)\right]}{\partial \tau_{2}} \\
&+\frac{\partial q_{N L}\left[\mathbf{y}\left(\tau_{1}, \tau_{2}, \tau_{3}\right)\right]}{\partial \tau_{3}}+i_{N L}\left[\mathbf{y}\left(\tau_{1}, \tau_{2}, \tau_{3}\right)\right] \\
&=\mathbf{x}\left(\tau_{1}, \tau_{2}, \tau_{3}\right) .
\end{aligned}
$$

Depending on the excitation regime determined by the RF carrier, the modulation envelope, and the LO signals, this MPDE can either be solved in the timedomain, frequency-domain, or a combination of both. Indeed, if any of the stimuli are periodic in some time variable, it is convenient to treat it in the frequencydomain using the Discrete Fourier Transform (DFT) and its inverse (IDFT). If a stimulus is aperiodic, then it should be kept in time-domain. That is, it is the nature of the excitation regimes that ultimately should determine the choice for a particular analysis method.

\section{A. Multidimensional HB Technique}

If the mixer were memoryless with respect to the RF envelope (mixer behavior is frequency-independent within the envelope bandwidth), then the circuit would handle the excitation as if the envelope had no bandwidth, or were periodic. In that case, $\mathbf{x}\left(\tau_{1}, \tau_{2}, \tau_{3}\right)$ can be approximated by one LO sinusoid at $\omega_{0}$ plus an $\mathrm{RF}$ carrier, at $\omega_{R F}$, modulated by another sinusoid at $\omega_{m}$.

Considering the eventual mixing products between these components, we would get a solution which can be expressed in a 3D frequency-domain as:

$$
\begin{aligned}
& \mathbf{y}\left(\tau_{1}, \tau_{2}, \tau_{3}\right) \\
& =\sum_{k_{1}=-K_{1}}^{K_{1}} \sum_{k_{2}=-K_{2}}^{K_{2}} \sum_{k_{3}=-K_{3}}^{K_{3}} \mathbf{Y}\left(k_{1}, k_{2}, k_{3}\right) e^{j\left(k_{1} \omega_{0} \tau_{1}+k_{2} \omega_{R F} \tau_{2}+k_{3} \omega_{m} \tau_{3}\right)}
\end{aligned}
$$

Substituting this 3D DFT form into the MPDE of Eq. (6), it becomes a multidimensional harmonic-balance equation (MDFT-HB):

$$
\begin{aligned}
j \Omega \mathbf{Q}_{n l}\left[\mathbf{Y}\left(k_{1}, k_{2}, k_{3}\right)\right]+\mathbf{I}_{n l}\left[\mathbf{Y}\left(k_{1}, k_{2}, k_{3}\right)\right] \\
=\mathbf{X}\left(k_{1}, k_{2}, k_{3}\right)
\end{aligned}
$$

in which $\boldsymbol{\Omega}$ is the frequency matrix.

Equation (8) can now be solved directly for the Fourier coefficients of $\mathbf{Y}\left(k_{1}, k_{2}, k_{3}\right)$ using a 3D harmonic-balance scheme [5]. However, as will be shown next, it can also be solved using a conventional 1D DFT-HB if the 3D spectrum is mapped onto an appropriate 1D artificial frequency-domain [6].

\section{B. Artificial Frequency Mapping HB Technique}

Artificial Frequency Mapping (AFM) was conceived to handle multidimensional $\mathrm{HB}$ problems with much simpler conventional HB solvers [2, 6, 14]. These techniques were applied earlier to modulated excitations when these were represented by evenly separated multitone spectra under diamond or box truncation schemes [3, 4]. Subsequently, they were extended to the simulation of mixers by incorporating the sinusoidal LO into the composite excitation, keeping the $\mathrm{RF}$ as a discrete uniformly separated multitone signal [6].

In this case, the RF signal is a sinusoidal carrier modulated by a general periodic envelope:

$$
x_{R F}\left(\tau_{2}, \tau_{3}\right)=r\left(\tau_{3}\right) \cos \left[\omega_{R F} \tau_{2}+\phi\left(\tau_{3}\right)\right]
$$

such that $x_{m}\left(\tau_{3}\right)$ is now given by:

$$
x_{m}\left(\tau_{3}\right)=r\left(\tau_{3}\right) e^{j \phi\left(\tau_{3}\right)}=\sum_{k_{3}=-K_{3}}^{K_{3}} X_{m}\left(k_{3}\right) e^{j k_{3} \omega_{m} \tau_{3}}
$$

with $K_{3}$ the maximum number of harmonics considered.

However, AFM techniques are not restricted to regimes where the $\mathrm{LO}$ and the RF spectra share the same truncation scheme. They can also be used when the excitation spectrum is more conveniently truncated in diamond form for the equally spaced multitone RF signal of small amplitude, and truncated in box form for the much stronger sinusoidal LO signal [6]. The algorithm necessary for the generation of such a mapped frequency index can be divided into two steps.

The first step consists of applying the AFM for diamond truncated spectra, previously developed for amplifiers [3, 4], to our equally spaced multitone RF input. This mapped spectrum, composed of a certain 


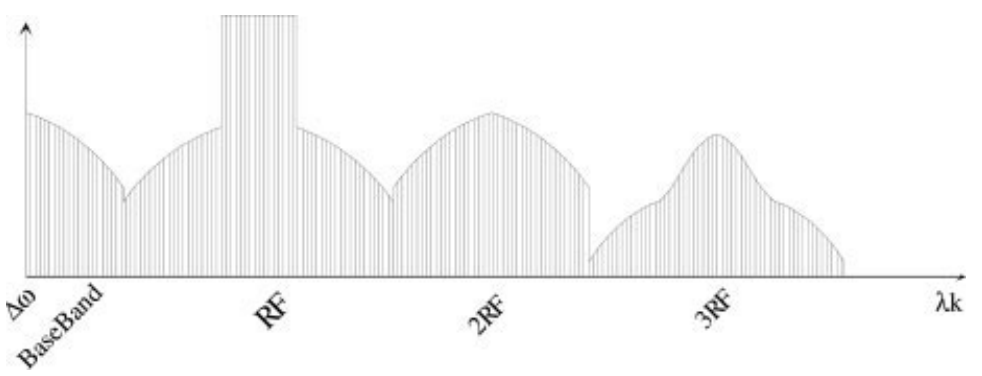

Figure 2. Output diamond truncated RF mapped spectrum.

number of adjacent clustered mixing products, is shown in Figure 2.

In the second step, this diamond truncated mapped spectrum is considered as a new composite tone to be mixed with the LO. The resulting mixing products are then box truncated, and another AFM is considered. As illustrated in Figure 3, the resulting spectrum corresponds to a dense and periodic frequency index vector which can readily be used in a conventional 1D HB engine.

Unfortunately, as Eq. (11) indicates, the total number of unknowns, $N$, rises quickly with the truncation orders of the RF and LO, $O_{R F}$ and $O_{L O}$, and the number of tones in each RF diamond truncation tone cluster, $n_{\text {diamond }}$ :

$$
\begin{aligned}
N \approx 2\left(\frac{O_{R F}+1}{2} O_{R F}+\right. & \left.\frac{O_{R F}}{2}\left(O_{R F}-1\right)\right) \\
& \cdot\left(1+2 O_{L O}\right) \cdot n_{\text {diamond }}
\end{aligned}
$$

This renders the AFM technique of limited practical use when the envelope spectrum demands discretization in a large number of tones (the envelope tends to be really an aperiodic signal), or when the mixer presents rather strong nonlinearities. In those cases it is preferable to keep the time-domain description of the envelope, and to solve the mixer equations for each of the envelope time-steps. In this way, a timemarching envelope simulation substitutes the solution of a single very complex steady-state problem by a succession of many, much simpler, time-step solutions.

\section{Multi-Envelope Transient Techniques Coupled with HB}

If $\mathbf{x}\left(\tau_{1}, \tau_{2}, \tau_{3}\right)$ is composed of a periodic LO (in $\tau_{1}$ ) and a periodic RF (in $\tau_{2}$ ) modulated by an aperiodic envelope (in $\tau_{3}$ ), then it can be represented as a time-varying 2D DFT:

$\mathbf{x}\left(\tau_{1}, \tau_{2}, \tau_{3}\right)=\sum_{k_{1}=-K_{1}}^{K_{1}} \sum_{k_{2}=-K_{2}}^{K_{2}} \mathbf{X}\left(k_{1}, k_{2}, \tau_{3}\right) e^{j\left(k_{1} \omega_{0} \tau_{1}+k_{2} \omega_{R F} \tau_{2}\right)}$

whose format will also be shared by all mixer state variables:

$\mathbf{y}\left(\tau_{1}, \tau_{2}, \tau_{3}\right)=\sum_{k_{1}=-K_{1}}^{K_{1}} \sum_{k_{2}=-K_{2}}^{K_{2}} \mathbf{Y}\left(k_{1}, k_{2}, \tau_{3}\right) e^{j\left(k_{1} \omega_{0} \tau_{1}+k_{2} \omega_{R F} \tau_{2}\right)}$

Substituting these $\tau_{3}$-varying 2D DFTs into the MPDE of Eq. (6), turns it into a 2D HB equation in $\left(k_{1} \omega_{0}, k_{2} \omega_{R F}\right)$, which is, simultaneously, an ODE in $\tau_{3}:$

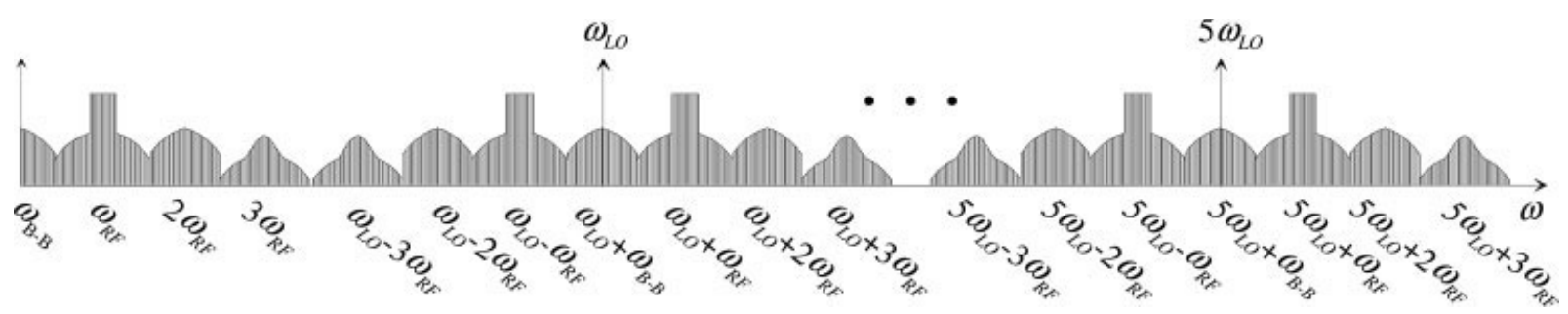

Figure 3. Final mapped spectrum obtained from a diamond truncation AFM to the low level RF signal, followed by a box truncation AFM for including the much stronger in amplitude periodic LO. 


$$
\begin{aligned}
\mathbf{j} \mathbf{\Omega Q}_{n}\left[\mathbf{Y}\left(k_{1}, k_{2}, \tau_{3}\right)\right]+\frac{\partial q_{N L}\left[\mathbf{Y}\left(k_{1}, k_{2}, \tau_{3}\right)\right]}{\partial \tau_{3}} \\
+\mathbf{I}_{n l}\left[\mathbf{Y}\left(k_{1}, k_{2}, \tau_{3}\right)\right]=\mathbf{X}\left(k_{1}, k_{2}, \tau_{3}\right)
\end{aligned}
$$

With a convenient $\tau_{3}$ time-discretization scheme, this envelope transient $\mathrm{HB}$ equation is solved for each of the envelope time-steps $\tau_{3}$.

\section{Time-Varying Volterra Series}

Unfortunately, all of the above methods are numerical in nature, not allowing for the extraction of a mixer model. They are, therefore, inappropriate for mixer design. An analysis technique capable of providing a suitable model for mixer design is the Volterra series method [1]. Unfortunately, since it is based on a Taylor expansion (around some predetermined quiescent point), its application becomes restricted to mildly nonlinear regimes. In mixer analysis, this means that the input RF and output IF signals must be considered as small perturbations of the much stronger time-varying quiescent point imposed by the LO voltage and any eventual dc bias. Alternatively, behavioral modeling techniques using statistical representations of signals and power series models of nonlinearities are still evolving [15]. They have not matured to the point where they can be applied to the general mixer problem being considered here.

This perturbed quiescent point, or mildly nonlinear assumption, allows a two-step procedure usually known as the large-signal/small-signal analysis $[1,2]$. First, the time-varying quiescent point is determined, using a true strongly nonlinear analysis. Then the method proceeds as a time-varying Volterra series analysis for the mild RF excitation and IF response.
In the first step, the mixer is analyzed under no RF excitation, for the dc bias and LO alone. $\mathbf{x}(t)$ is thus only dependent on $\tau_{1}$ and the MPDE (this way converted into an ODE in $\tau_{1}$ ) admits the following periodic solution:

$$
\mathbf{y}\left(\tau_{1}\right)=\sum_{k_{1}=-K_{1}}^{K_{1}} \mathbf{Y}\left(k_{1}\right) e^{j k_{1} \omega_{0} \tau_{1}}
$$

The substitution of this form into the MPDE leads to a conventional sinusoidal HB equation:

$$
\mathbf{F}\left[\mathbf{Y}\left(k_{1}\right)\right] \equiv \mathbf{j} \boldsymbol{\Omega} \mathbf{Q}_{n l}\left[\mathbf{Y}\left(k_{1}\right)\right]+\mathbf{I}_{n l}\left[\mathbf{Y}\left(k_{1}\right)\right]-\mathbf{X}\left(k_{1}\right)=0
$$

whose solution in time, $\mathbf{y}\left(\tau_{1}\right)$, allows for the determination of all $\tau_{1}$-dependent algebraic nonlinearities, $q_{N L}\left[\mathbf{y}\left(\tau_{1}\right)\right]$ and $i_{N L}\left[\mathbf{y}\left(\tau_{1}\right)\right]$.

In the second step, the mixer LO response is perturbed by the RF input. Assuming the RF signal varies along $\tau_{2}$ and is a small perturbation of the $\tau_{1}$-varying quiescent point, nonlinearities can be expanded in a Taylor series. For example, for the current nonlinearity we have:

$$
\begin{aligned}
i_{N L}\left[\mathbf{y}\left(\tau_{1}, \tau_{2}\right)\right] \approx g_{1}\left(\tau_{1}\right) \mathbf{y}\left(\tau_{1}, \tau_{2}\right) & +g_{2}\left(\tau_{1}\right) \mathbf{y}\left(\tau_{1}, \tau_{2}\right)^{2} \\
& +g_{3}\left(\tau_{1}\right) \mathbf{y}\left(\tau_{1}, \tau_{2}\right)^{3}
\end{aligned}
$$

Then the time-domain products, e.g., $g_{1}\left(\tau_{1}\right) \mathbf{y}\left(\tau_{1}, \tau_{2}\right)$, are converted into frequency-domain convolutions, $G_{1}\left(k_{1}\right) * \mathbf{Y}\left(k_{1}, k_{2}\right)$. These are more efficiently evaluated as products of a Toeplitz matrix - the conversion matrix- $G_{1}\left(k_{1}\right)$ by a vector $\mathbf{Y}\left(k_{1}, k_{2}\right)$.

Using the conversion matrix formulation, the mixer circuit can be modeled as a set of $\tau_{1}$-varying Volterra nonlinear transfer functions, $\mathbf{H}_{n}\left(\tau_{1}, \omega_{1}, \ldots, \omega_{n}\right)$ :

$$
\begin{aligned}
\mathbf{y}\left(\tau_{1}, \tau_{2}\right)=\int_{-\infty}^{\infty} \mathbf{H}_{1}\left(\tau_{1}, \omega\right) \mathbf{X}\left(\tau_{1}, \omega\right) e^{j \omega \tau_{2}} d \omega+\int_{-\infty}^{\infty} \int_{-\infty}^{\infty} \mathbf{H}_{2}\left(\tau_{1}, \omega_{1}, \omega_{2}\right) \mathbf{X}\left(\tau_{1}, \omega_{1}\right) \mathbf{X}\left(\tau_{1}, \omega_{2}\right) e^{j\left(\omega_{1}+\omega_{2}\right) \tau_{2}} d \omega_{1} d \omega_{2} \\
+\int_{-\infty}^{\infty} \int_{-\infty}^{\infty} \int_{-\infty}^{\infty} \mathbf{H}_{3}\left(\tau_{1}, \omega_{1}, \omega_{2}, \omega_{3}\right) \cdot \mathbf{X}\left(\tau_{1}, \omega_{1}\right) \mathbf{X}\left(\tau_{1}, \omega_{2}\right) \mathbf{X}\left(\tau_{1}, \omega_{3}\right) e^{j\left(\omega_{1}+\omega_{2}+\omega_{3}\right) \tau_{2}} d \omega_{1} d \omega_{2} d \omega_{3}
\end{aligned}
$$

which constitutes the desired analytic mildly nonlinear model of the mixer.

Although valid for both mixer analysis and design, under periodic or aperiodic RF excitations, the above frequency-domain Volterra model is restricted to periodic LOs and mildly nonlinear regimes. Therefore, it leaves unresolved a significant group of important mixer simulation problems such as modeling the cir- 


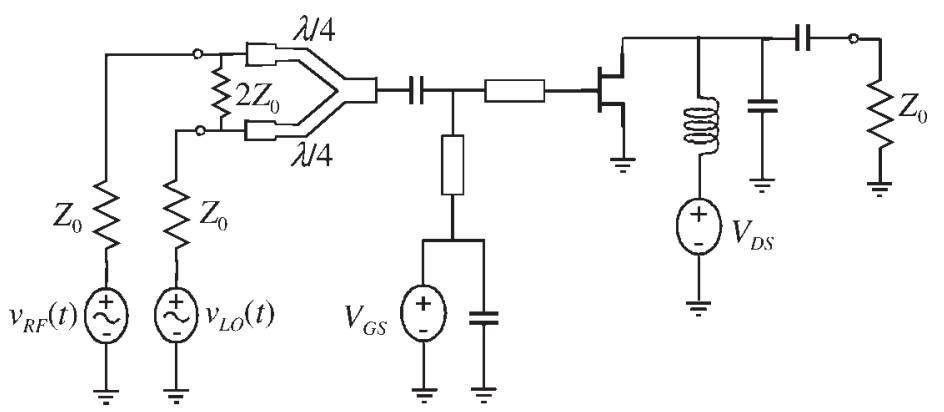

Figure 4. Active MESFET mixer example used in the simulation experiments.

cuit's $1 \mathrm{~dB}$ compression point and power saturation, the desensitization caused by a strong interferer, or even LO phase noise effects.

\section{APPLICATION EXAMPLES}

To illustrate the application to system level performance of some of the mixer simulation techniques presented above, consider the real mixer model of Figure 4. It is a simplified version of a GaAs MESFET-based active mixer in which both the LO and RF signals are applied to the transistor gate. In the present case, the input diplexer is a simple Wilkinson coupler followed by broadband matching and biasing networks. The resulting IF is collected at the MESFET drain and passed through a bypass capacitor to the resistive load. Note that the drain inductor behaves as an RF choke to the IF, while the capacitor to ground is a short for both the RF and LO frequencies, but an open-circuit to the desired IF. Such a mixer circuit is typically biased at turn-on and in the MESFET's saturation region for improved RF-IF conversion efficiency.

Although two-tone HB simulation-either based on MDFT or an AFM technique-is now the most common mixer simulation scheme, it is being progressively substituted by more advanced methods. In fact, since it assumes sinusoidal RF and LO excitation, the zero-bandwidth restriction imposed on the RF signal obviates any study of the dynamic impairment on the envelope, and thus on the transmitted information. Extensions of this two-tone $\mathrm{HB}$ have also been applied to mixer distortion analysis to accommodate two RF tones (time-varying Volterra series or 3D DFT based $\mathrm{HB}$ ) [2], and also multitone RF signals (multitone AFM techniques for mixer analysis) $[2,6]$. Although this technique was conceived of for circuit simulation, it cannot be used at the system analysis level.
Since envelope transient techniques directly handle the complex envelope, they can be easily extended to system analysis if two convenient system/circuit and circuit/system interfaces are provided. As depicted in Figure 5, these interfaces build the corresponding RF modulated signal from the input information content, and then convert the modulated IF back into the information level.

\section{A. Modulated RF Signal}

As a starting point, we begin a simple illustrative example of a mixer excited by a modulated RF signal whose spectrum (specifically, the Power Spectral Density function, PSD) is depicted in Figure 6 (where the plotted curve refers to the output power calculated in a $1 \mathrm{~Hz}$ resolution bandwidth). It consists of a $2 \mathrm{GHz}$ carrier that is down-converted to an IF of $100 \mathrm{MHz}$ by a pure sinusoidal LO of $2.1 \mathrm{GHz}$.

Figures 7 and 8, respectively, represent the frequency-domain PSD of the resulting IF and its timedomain in-phase and quadrature complex-envelope waveform components. Comparing the input and output PSDs, it can be concluded that this represents the ideal mixer operation in which the output PSD is simply a down-converted linear replica of the input. This is a consequence of small-signal linear operation. So, this simulation example could even be efficiently handled by a conventional small-signal/large-signal analysis.

\section{B. Image Frequency Interference}

Another practical example of mixer simulation refers to the study of the impact of an interfering channel present at the image frequency (IM). As depicted in Figure 9, the IM is, in the present case, located at $\omega_{O}+\omega_{I F}=2 \omega_{0}-\omega_{R F}$. Depending on the level of the RF and IM signals, this can, or can no longer, be handled by a linear analysis. However, it can still be treated by 


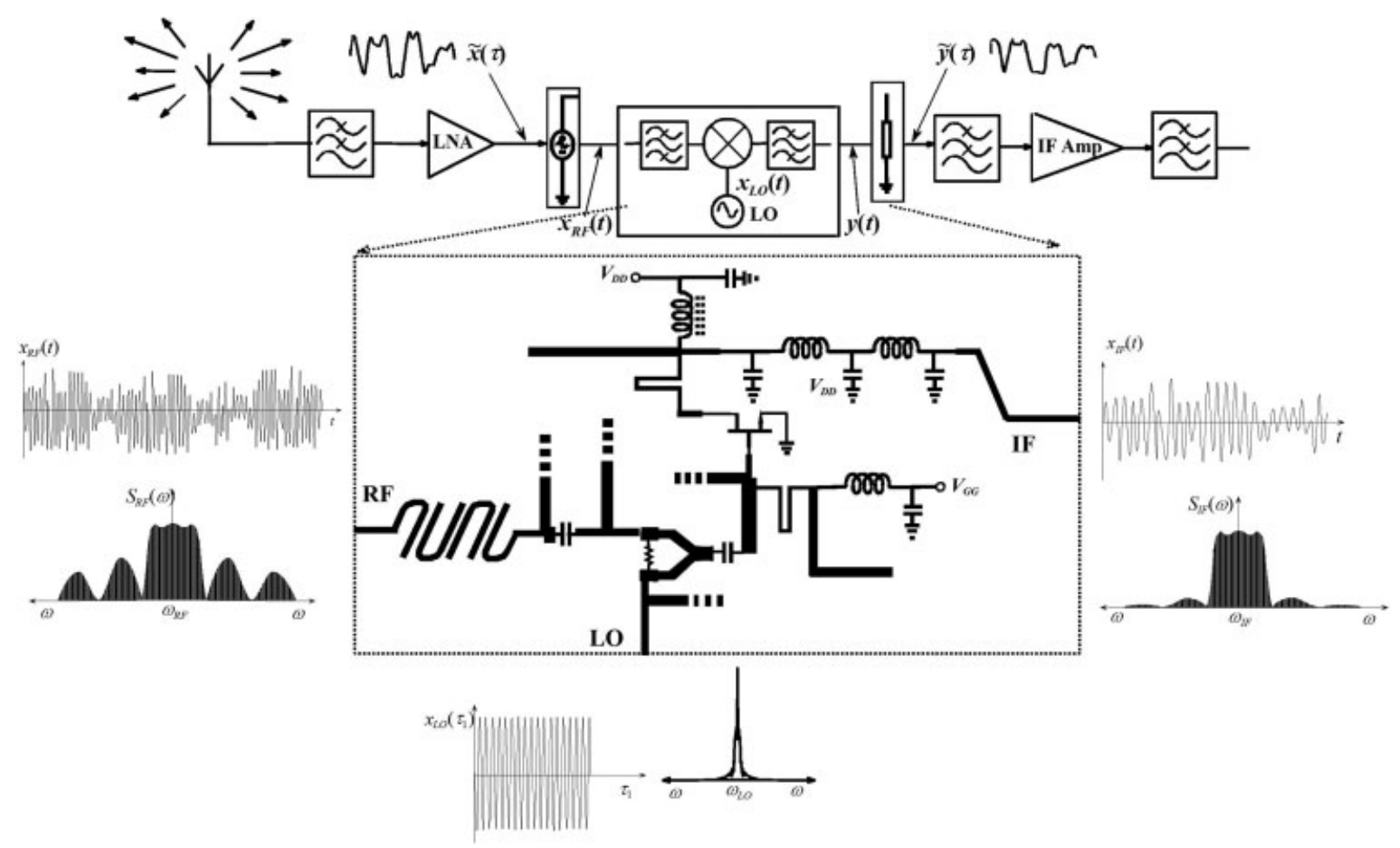

Figure 5. Example of system simulation of a microwave mixer using the envelope transient technique and appropriate system/circuit/system signal interfaces. Note that only the mixer circuit handles the complete modulated RF signals; all other system components process either the information content or the complex base-band envelope.

an envelope driven simulator if three $\mathrm{HB}$ tones (for the RF, IM, and LO sinusoids) and two envelope time-scales (associated with the RF and IM carriers) are considered.

Taking the RF and LO signals, already considered for our reference example, and the IM channel PSD shown in Figure 10, the perturbed IF output PSD is as plotted in Figure 11.

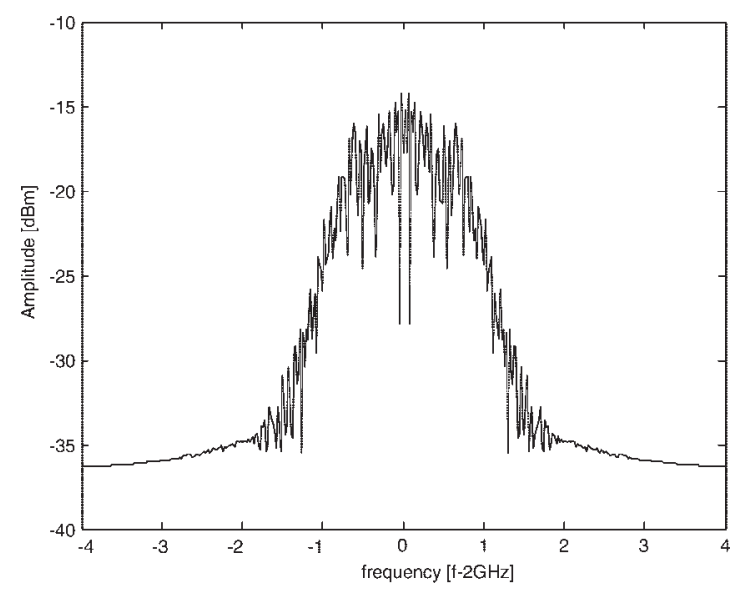

Figure 6. Input power spectral density function of the RF mixer stimulus used in the simulation examples.
A comparison of Figures 7 and 11 reveals the notorious spectrum widening effect caused by the IM channel, as its small amplitude condition determined a linear additive noise operation.

Having kept the small-signal condition for both the $\mathrm{RF}$ and the IM signals, this simulation example could still be efficiently handled by a conventional smallsignal/large-signal analysis.

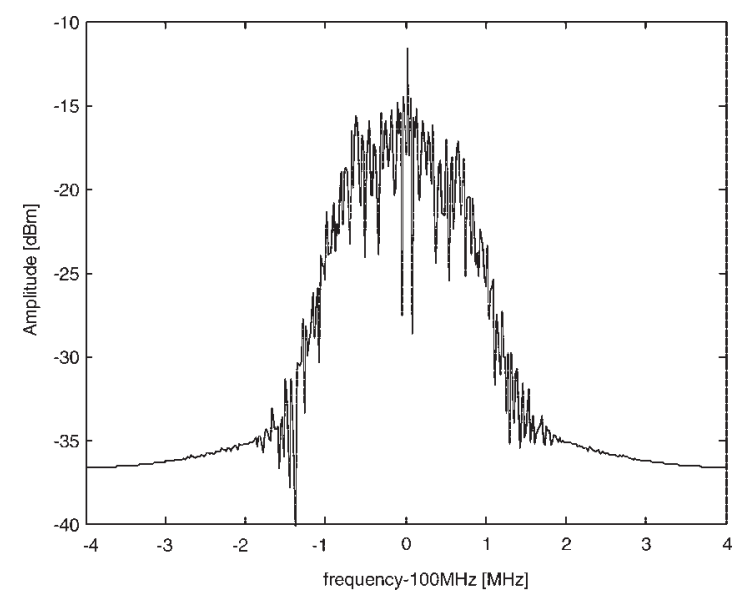

Figure 7. Output IF power spectral density function resulting from the PSD stimulus depicted in Figure 6. 


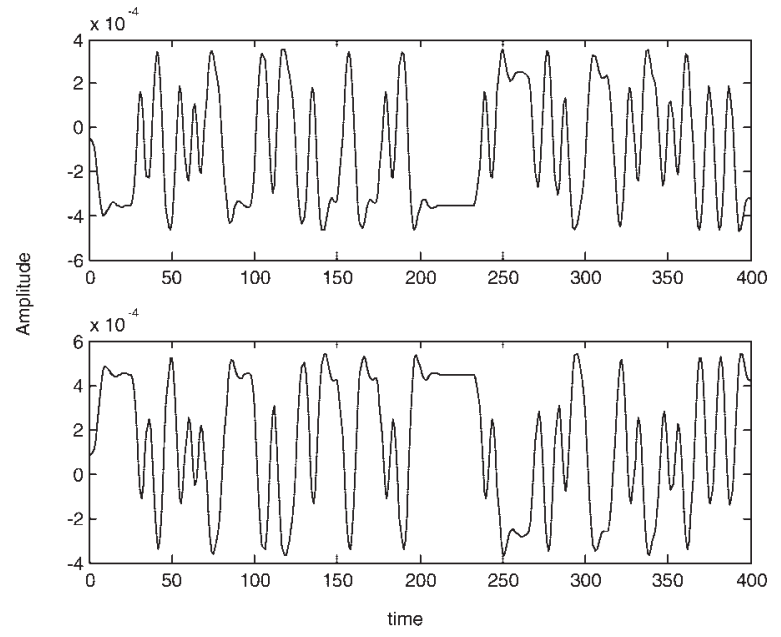

Figure 8. Time-domain waveforms of the in-phase and quadrature output IF complex envelope correspondent to the spectrum shown in Figure 7.

System designers most often face a slightly different, but usually more relevant, situation. This is the case where the interferer comes no longer through the image frequency band (in well-designed systems protected by an image-rejection filter), but is closely located to the desired RF signal. Although a conventional linear mixer analysis would lead to the conclusion that such an adjacent channel had no impact on the RF-IF conversion process, the situation is dramatically different if nonlinearity is added to the mixer. That is, if the interferer has such a strong amplitude that it is capable of exciting the mixer nonlinearities, then it not only will convert itself to the IF band, but also it is capable of reducing the conversion gain to the RF signal.

In fact, nonlinear analysis [2] shows that a timevarying third-order nonlinearity excited by two signals (beyond the $\mathrm{LO}$ ), at $\omega_{R F}$ with amplitude $A_{R F}$, and at the adjacent channel at $\omega_{A C}$ with amplitude $A_{A C}$, will produce the linear output IF at $\omega_{I F}=\omega_{0}-\omega_{\mathrm{RF}}$, with an amplitude that is proportional to $A_{R F}$, a linear output at $\omega_{0}-\omega_{\mathrm{AC}}$ proportional to $A_{A C}$ but also a nonlinear IF mixing product of frequency $\omega_{I F}=\omega_{0}-$

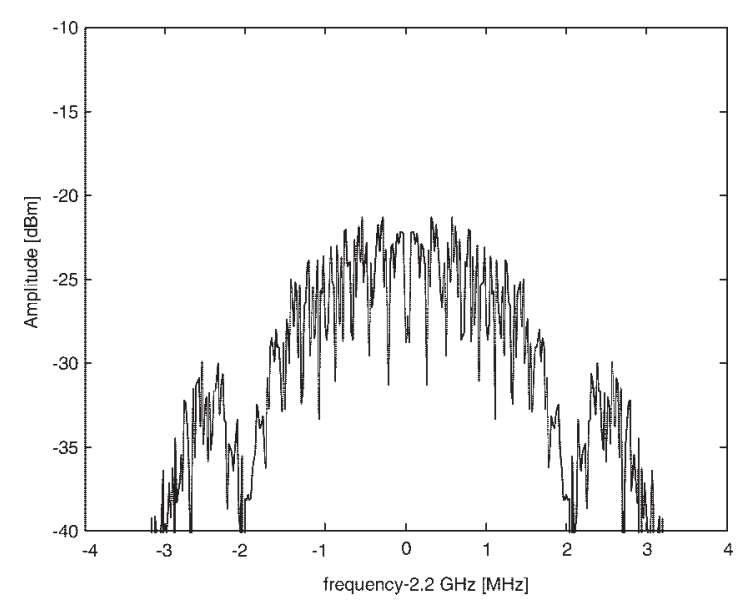

Figure 10. Input power spectral density function of the interferer signal applied at the image frequency band.

$\omega_{\mathrm{RF}}+\omega_{\mathrm{AC}}-\omega_{\mathrm{AC}}$ whose amplitude is proportional to $A_{R F} \cdot A_{A C}$. For sinusoidal interferers, composed of a single spectral component, the $\omega_{A C}-\omega_{A C}$ term is always zero. So, as a result of the mixer gain compression characteristics, this nonlinear mixing product, in general, implies a reduction of mixer conversion gain. However, for modulated interferers, composed of various spectral components, where $\omega_{A C_{i}}$ - $\omega_{A C_{j}}$ (where $\omega_{A C_{i}}$ and $\omega_{A C_{j}}$ stand for any two different frequency components of the $\omega_{A C}$ excitation set) ranges from zero up to the bandwidth of the interferer, this mixing product also adds a cross modulated effect that can be interpreted as a conversion of the interferer to the desired IF band.

Unfortunately, a conventional linear mixer analysis can only deal with weak adjacent channel interferers, which, falling outside the IF band, cause no impact on system performance. The situation of practical interest demands a complete nonlinear analysis like the one illustrated by the simulation example of Figure 12. Here the same RF and LO signals are assumed as before, but now a strong adjacent channel has been located at $2.05 \mathrm{GHz}$. The resulting IF is shown in Figure 13, which, compared to our IF reference of

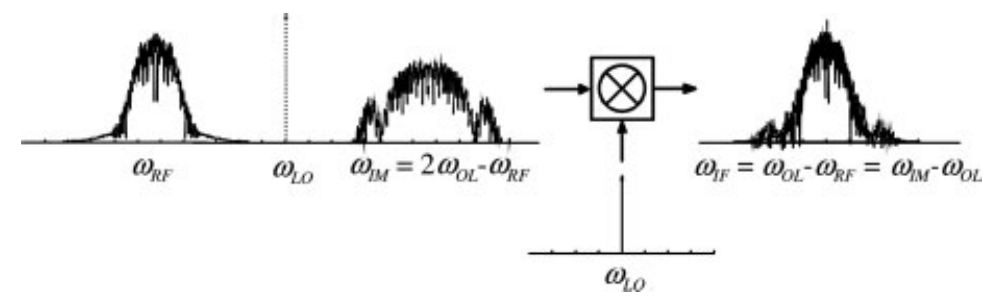

Figure 9. Illustrative mixer simulation in which the RF is a modulated signal and the LO is a sinusoidal carrier, but there is also an interfering modulated signal at the mixer IM band. 


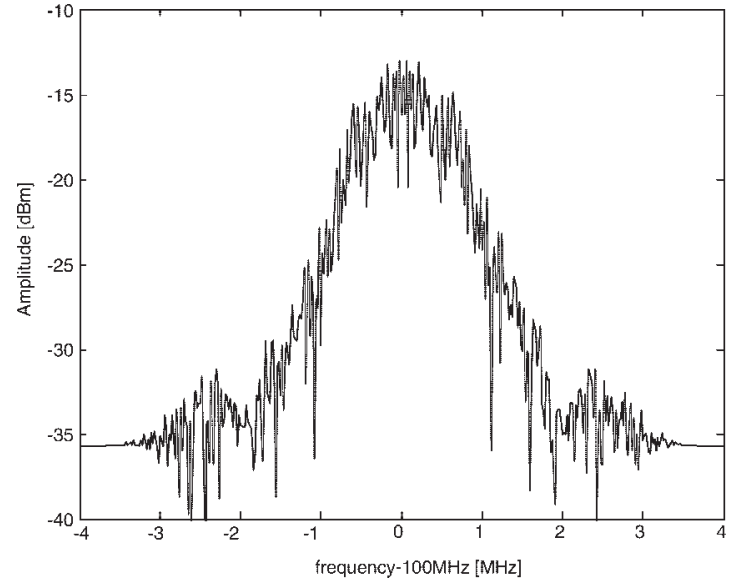

Figure 11. Output IF power spectral density function resulting from the stimulus RF and the IM interferer shown in Figures 6 and 10, respectively.

Figure 7 , clearly reveals the theoretically predicted signal perturbation.

\section{AC Interferer and LO Phase Noise}

As a final example, we will discuss a very important situation for system designers which, being highly demanding in terms of the simulation algorithm, is, most of the time, left untreated. It is the case illustrated by Figure 14 of a mixer excited by the RF signal, a weak or strong $\mathrm{AC}$ interferer, and a local oscillator corrupted by a certain amount of phase noise.

Here the RF signal considered was as described before, the adjacent channel interferer was similar to the one of Figure 10 but backed off in power by 20 $\mathrm{dB}$, and the PSD of the new LO signal is shown in Figure 15.

Beyond the perturbation effects already studied, there are two new ones that can be directly linked to the LO phase noise: 1) contamination of the IF by LO noise; and 2) the effect of an AC interferer.

The first effect is attributable to the linear mixing process, and results in the contamination of the IF by the LO noise. Keeping in mind that frequency and

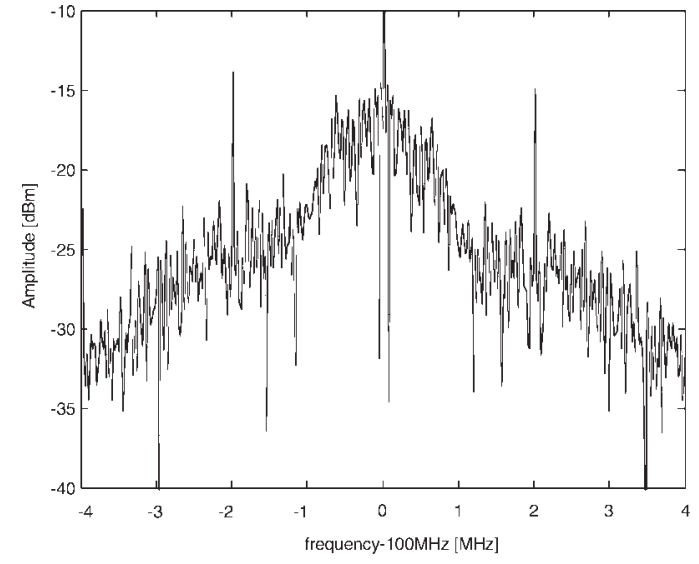

Figure 13. Output IF power spectral density function resulting from the mixer simulation example illustrated in Figure 12.

phase are directly related by the differential operator, we conclude that phase noise also implies frequency noise. So, if the LO frequency is actually $\omega_{0}(t)=\omega_{0}$ $+\Omega(t)$, then the resulting $\operatorname{IF}\left(\omega_{I F}(t)=\omega_{0}(t)-\omega_{\mathrm{RF}}\right)$ will necessarily be also corrupted by phase noise.

Seen in the frequency-domain, this corresponds to widening of the IF PSDs due to the RF signal and the adjacent channel interferer. To understand this we must realize that, as seen in Figure 15, a LO with phase noise no longer comprises just a single frequency component at $\omega_{0}$, but can be understood as a cluster of many densely located tones around the original $\omega_{0}$. If we now consider all mixing processes induced by each of these LO tones, we immediately conclude that the resulting IF will be the sum of each of those IFs. Therefore, the total IF bandwidth for the RF will be the RF bandwidth plus the LO spectral line width. This represents a redistribution of RF signal power throughout this new IF bandwidth, which will be afterwards turned into wasted signal power when the spectrum is clipped by the abrupt IF channel selection filter.

The second effect is that of the interferer, which can now be readily understood. Since its correspond-

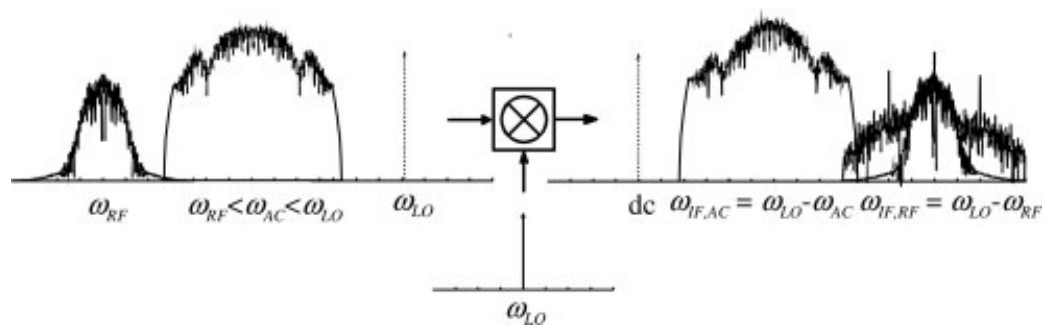

Figure 12. Illustrative mixer simulation in which the LO is a sinusoidal carrier and the RF is a modulated signal accompanied by another closely located channel of much stronger amplitude. 


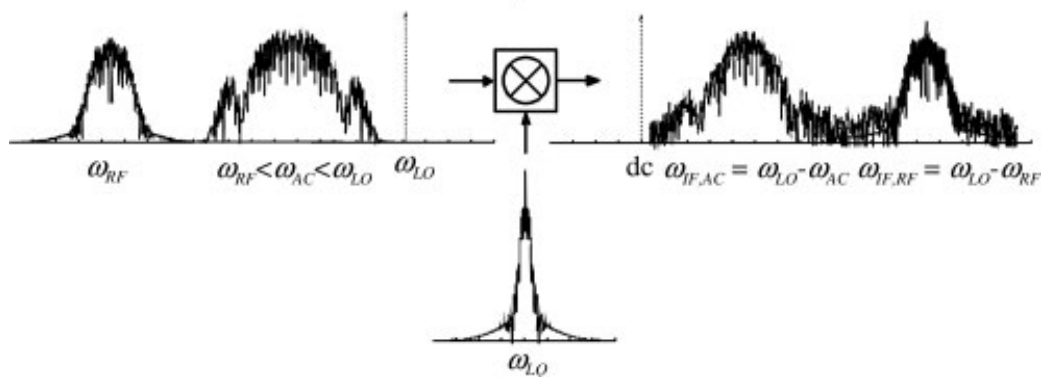

Figure 14. Mixer simulation when the RF is a modulated signal and the LO is a sinusoidal carrier corrupted by phase noise, but there is also an interferer modulated signal component at the input of the system.

ing IF will also be widened, it can constitute important interference whenever the RF and the AC IFs overlap. This begins to happen for $\mathrm{AC}$ interferers whose central frequencies differ less than one-half of the sum of the LO, RF, and AC bandwidths from the central frequency of the desired RF channel. In modern wireless systems, where the band guards have been reduced to nearly zero, this effect can constitute severe signal impairment.

As has been stated, the simulation of such effects is not easy. Note that in spite of dealing with a conventional time-varying linear process, it is already clear that the situation is beyond the conventional conversion-matrix based small-signal/large-signal analysis, as the LO regime is no longer periodic. In fact, the stochastic nature of the LO phase noise prevents the use of any frequency-domain technique.

This problem, however, can be circumvented if the LO signal is considered as a periodic carrier modulated in phase by noise. In this case, analysis requires a multirate regime composed of six independent time scales for the LO, RF, and AC carriers, plus their

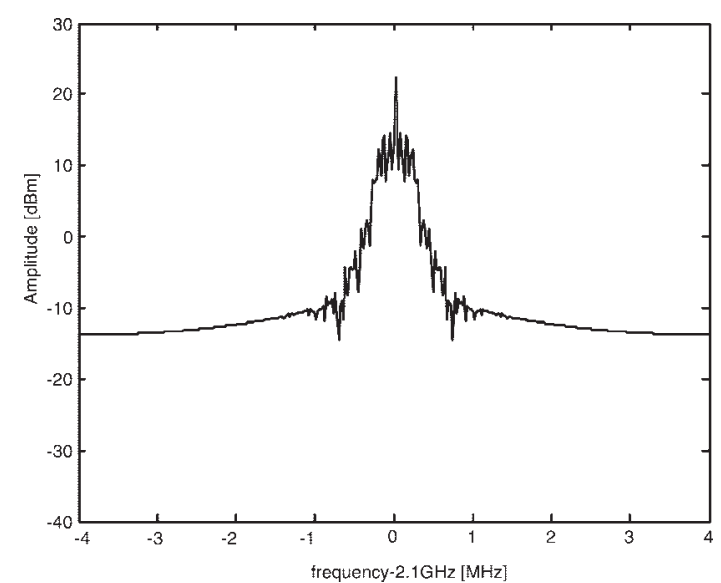

Figure 15. Spectrum of LO signal corrupted by phase noise. three modulation envelopes. This approach was followed here to obtain the RF and AC output IF PSD results shown in Figures 16 and 17, respectively. Benefiting from the periodic character of the three carriers and the stochastic nature of their envelopes, a time-step integration was conducted for the complex envelopes, over a three-tone AFM-based HB. Through this approach, different simulation conditions can be accommodated ranging from the linear periodic regime to the truly nonlinear stochastic regime in which any or all of three modulated signals can excite the mixer MESFET nonlinearities.

\section{CONCLUSIONS}

In this article an overview of various mixer simulation techniques, capable of providing relevant information for system level design, was presented. It was demonstrated that it is not possible to rely on conventional black-box models to conduct accurate nonlinear and

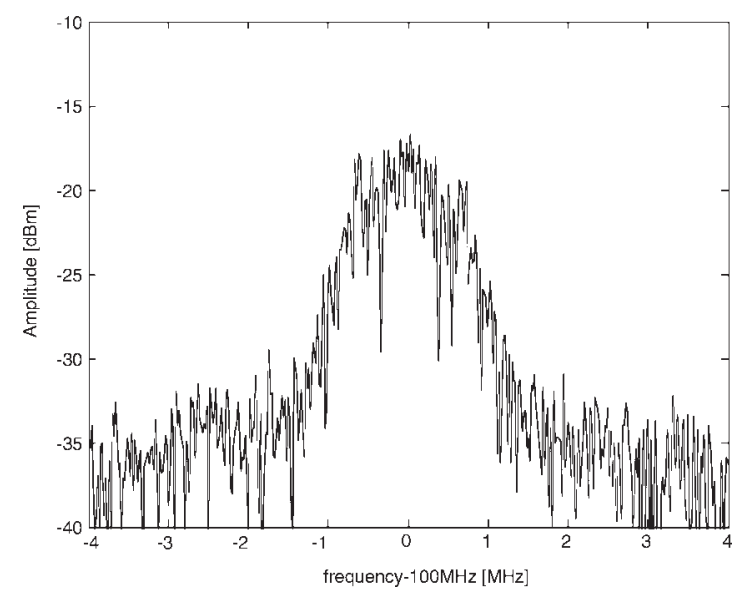

Figure 16. Output IF power spectral density function due to the RF input, resulting from the mixer simulation example illustrated in Figure 14. 


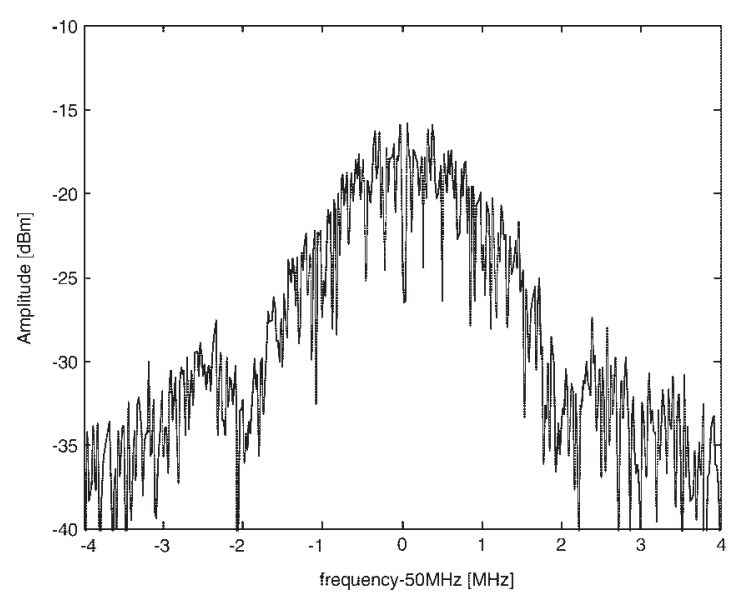

Figure 17. Output IF power spectral density function due to the adjacent channel interferer, resulting from the mixer simulation example illustrated in Figure 14.

dynamic mixer analysis. Thus, circuit level simulation still constitutes a reliable alternative.

Harmonic-balance, either based on MDFT or AFM, is appropriate to periodic or quasi-periodic $\mathrm{LO}$ and $\mathrm{RF}$ stimulus, but presents difficulties with truly aperiodic RF envelopes. On the other hand, multi-envelope techniques seem to be especially useful in treating aperiodic regimes of either single or multicarrier RF inputs and LO corrupted by phase noise. In addition, these multi-envelope techniques are also important to bridge circuit level and system level simulation.

Nevertheless, as circuit and system level design requires a closed form mixer model, a Volterra series analysis must be undertaken. Unfortunately, with Volterra analysis the RF is restricted to be a small perturbation of the time-varying LO quiescent point, which then needs full nonlinear simulation by an $\mathrm{HB}$ or envelope transient engine.

In summary, due to current limitations in behavioral modeling, designers of systems with mixers are currently compelled to start with a mildly nonlinear timevarying Volterra series analysis. This must then be complemented at the circuit level by a truly nonlinear and dynamic algorithm. For that, multi-envelope transient engines coupled with efficient AFM based HB machines constitute a powerful analysis tool.

\section{Acknowledgments}

N. B. Carvalho and J. C. Pedro acknowledge support by the European Community under the NoE TARGET. W. Jang and M. B. Steer acknowledge support by the U.S. Army Research Laboratory and the U.S. Army Research Office as a multidisciplinary University Research Initiative on Mul- tifunctional Adaptive Radio Radar and Sensors (MARRS) under grant number DAAD19-01-1-0496.

\section{REFERENCES}

1. S. Maas, Nonlinear microwave circuits, Artech House, Norwood, MA (1988).

2. J.C. Pedro and N.B. Carvalho, Intermodulation distortion in microwave and wireless circuits, Artech House, Norwood, MA (2003).

3. N.B. Carvalho and J.C. Pedro, Multi-tone frequency domain simulation of nonlinear circuits in large and small signal regimes, IEEE Trans Microwave Theory Tech, MTT-46 (1999), 2016-2024.

4. J.C. Pedro and N.B. Carvalho, Efficient harmonic balance computation of microwave circuits' response to multi-tone spectra, 29th European Microwave Conference Proc, Munchen, vol. I (Oct. 1999), 103-106.

5. V. Rizzoli, C. Cecchetti, and A. Lipparini, A generalpurpose program for the analysis of nonlinear microwave circuits under multi-tone excitation by multidimensional Fourier transform, 17th European Microwave Conference Proc, Rome (1987), 635-640.

6. N.B. Carvalho and J.C. Pedro, Novel artificial frequency mapping techniques for multi-tone simulation of mixers, 2001 IEEE Intern Microwave Theory Tech Symposium Dig, Phoenix (May 2001), 455-458.

7. E. Ngoya, J. Sombrin, and J. Rousset, Simulation des Circuits et Systèmes: Méthodes Actuelles et Tendances, in Séminaires Antennes Actives et MMIC, Arles, France (Apr. 1994), 171-176.

8. D. Sharrit, Private communication (June 1996).

9. V. Rizzoli, A. Neri, and F. Mastri, A modulationoriented piecewise harmonic-balance technique suitable for transient analysis and digitally modulated signals, 26th European Microwave Conference Proc, Prague (Sept. 1996), 546-550.

10. H. Brachtendorf, G. Welsch, R. Laur, and A. BunseGerstner, Numerical steady-state analysis of electronic circuits driven by multi-tone signals, Elec Eng, 79 (1996), 103-112.

11. J. Roychowdhury, Analysing circuits with widely separated time scales using numerical PDE methods, IEEE Trans Circuits Systems-I, 48 (2001), 578-594.

12. V. Rizzoli, C. Cecchetti, and A. Lipparini, A generalpurpose program for the analysis of nonlinear microwave circuits under multitone excitation by multidimensional Fourier transform, 17th European Microwave Conference Proc, Rome (Sept. 1987), 635-640.

13. Agilent Technologies, Advanced design system, Palo Alto, CA, 2002.

14. P. Rodrigues, Computer aided analysis of nonlinear microwave circuits, Artech House, Norwood, MA (1998).

15. K. Gard, H. Gutierrez, and M.B. Steer, Characterization of spectral regrowth in microwave amplifiers based on the nonlinear transformation of a complex gaussian process, IEEE Trans Microwave Theory Techn, 47 (1999), 1059-1069. 


\section{BIOGRAPHIES}

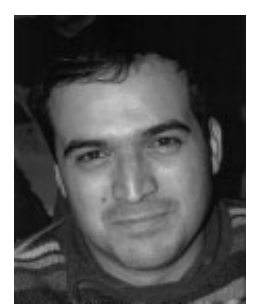

Nuno Borges Carvalho was born in Luanda, Portugal, in 1972. He received the diploma and doctoral degrees in Electronics and Telecommunications Engineering from the University of Aveiro, Aveiro, Portugal in 1995 and 2000, respectively. From 1997 to 2000 he was an Assistant Lecturer and a Professor from 2000. Currently he is an Associate Professor at the same university and a senior research scientist at the Telecommunications Institute. He has worked as a scientist researcher at the Telecommunications Institute and was engaged in different projects on nonlinear CAD and circuits. His main research interests include CAD for nonlinear circuits and design of RF-microwave power amplifiers. Dr. Carvalho is a member of the Portuguese Engineering Association and an IEEE member. He was the recipient of the 1995 University of Aveiro and the Portuguese Engineering Association Prize for the best 1995 student at the Universidade de Aveiro, the 1998 Student Paper Competition (third place) presented at the IEEE International Microwave Symposium, and the 2000 IEE Measurement Prize. He has been a reviewer for several magazines and is a member of the IEEE Transactions on Microwave Theory and Techniques Reviewer Board. Dr. Carvalho is co-author of the book Intermodulation in Microwave and Wireless Circuits from Artech House, 2003.

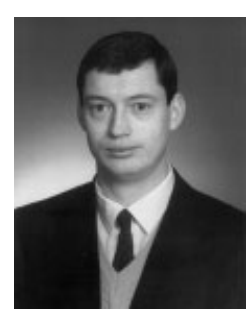

José C. Pedro was born in Espinho, Portugal, in 1962. He received the diploma, doctoral, and habilitation degrees in electronics and telecommunications engineering from the University of Aveiro, Portugal, in 1985, 1993, and 2002, respectively. From 1985 to 1993 he was an Assistant Lecturer at University of Aveiro, and a Professor since 1993. Currently he is a Professor at the same university, and a Senior Research Scientist at the Institute of Telecommunications. His main scientific interests include active device modeling and the analysis and design of various nonlinear microwave and optoelectronics circuits, in particular, the design of highly linear multicarrier power amplifiers and mixers. Dr. Pedro is the leading author of Intermodulation Distortion in Microwave and Wireless Circuits (Artech House, 2003), has authored or co-au- thored several articles in international journals and symposia, and served the IEEE as a reviewer for the MTT Transactions and the MTT-IMS. Dr. Pedro received the Marconi Young Scientist Award in 1993 and the 2000 Institution of Electrical Engineers (IEE) Measurement Prize.

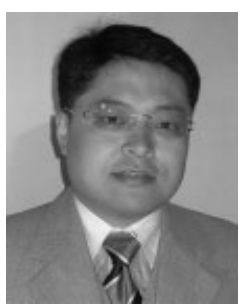

Wonhoon Jang received the B.S. degree in electronics from Kyungpook National University in Daegu, Korea, in 1997. He is presently working toward a Ph.D. degree in electrical engineering at North Carolina State University, Raleigh. From 1997 to 1999 he was with LG Precision Co., Kumi, Korea, where he worked on military radios. His current research interests include nonlinear RF/microwave system analysis and modeling.

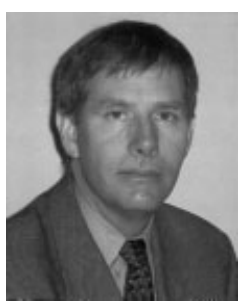

Michael Steer received his B.E. and Ph.D. in Electrical Engineering from the University of Queensland, Brisbane, Australia, in 1976 and 1983, respectively. Currently he is Professor of Electrical and Computer Engineering at North Carolina State University, Raleigh, NC. Professor Steer is a Fellow of the Institute of Electrical and Electronic Engineers for contributions to the computer aided engineering of nonlinear microwave and millimeter-wave circuits. $\mathrm{He}$ is active in the Microwave Theory and Techniques (MTT) Society. In 1997 he was Secretary of the Society and from 1998 to 2000 was an Elected Member of its Administrative Committee. In 1999 and 2000 he was Professor in the School of Electronic and Electrical Engineering at the University of Leeds, where he held the Chair in Microwave and Millimeterwave Electronics. He was also Director of the Institute of Microwaves and Photonics at the University of Leeds. He has authored around 300 publications on topics related to RF, microwave, and millimeter-wave systems, to highspeed digital design, and to RF and microwave design methodology and circuit simulation. He is co-author of the book Foundations of Interconnect and Microstrip Design, John Wiley \& Sons, 2000. Professor Steer is Editor-In-Chief of the IEEE Transactions on Microwave Theory and Techniques. 